\title{
Spin-Flipping a Stored Polarized Proton Beam with an rf Dipole*
}

\author{
B.B. Blinov ${ }^{\dagger}$, Ya.S. Derbenev, T. Kageya, D.Yu. Kantsyrev \\ A.D. Krisch, V.S. Morozov ${ }^{\ddagger}$, D.W. Sivers ${ }^{\S}$ and V.K. Wong \\ Randall Laboratory of Physics, University of Michigan, \\ Ann Arbor, Michigan 48109-1120 \\ V.A. Anferov, P. Schwandt and B. von Przewoski \\ Indiana University Cyclotron Facility Bloomington, Indiana 47408-0768
}

\begin{abstract}
Frequent polarization reversals, or spin-flips, of a stored polarized highenergy beam may greatly reduce systematic errors of spin asymmetry measurements in a scattering asymmetry experiment. We studied the spin-flipping of a $120 \mathrm{MeV}$ horizontally-polarized proton beam stored in the IUCF Cooler Ring by ramping an rfdipole magnet's frequency through an rf-induced depolarizing resonance in the presence of a nearly-full Siberian snake. After optimizing the frequency ramp parameters, we used multiple spin-flips to measure a spin-flip efficiency of $86.5 \pm 0.5 \%$. The spin-flip efficiency was apparently limited by the rf-dipole's field strength. This result indicates that an efficient spin-flipping a stored polarized beam should be possible in high energy rings such as RHIC and HERA where Siberian snakes are certainly needed and only dipole rf-flipper-magnets are practical.
\end{abstract}

\section{INTRODUCTION}

In a circular accelerator, each proton's spin precesses around the vertical magnetic fields of the ring's bending dipoles, with a frequency called the spin precession frequency $f_{s}$ that is related to the proton's circulation frequency $f_{c}$ by:

$$
f_{s}=f_{c} \nu_{s}
$$

where $\nu_{s}$ is the spin tune, which is the number of spin precessions during each turn around the ring.

*) Supported by grants from the U.S. Department of Energy and National Science Foundation.

†) Corresponding author.

$\ddagger$ also at Moscow State University, Moscow, Russia

§) also at Portland Physics Institute, Portland, Oregon 97201.

CP549, Intersections of Particle and Nuclear Physics: $7^{\text {th }}$ Conference, edited by Z. Parsa and W. J. Marciano (C) 2000 American Institute of Physics 1-56396-978-5/00/\$17.00 
If there are only vertical magnetic fields, then the vertical beam polarization remains unchanged; however, whenever there is a periodic horizontal magnetic field whose tune is equal to the spin tune a depolarizing resonance occurs, which can destroy the polarization. Since the $\nu_{s}$ is proportional to the proton's energy via:

$$
\nu_{s}=G \gamma
$$

where $G=1.792847$ is the proton's anomalous magnetic moment, and $\gamma$ is the Lorentz energy factor, the protons will encounter many depolarizing resonances as they are accelerated to a high energy.

An elegant method to overcome these depolarizing resonances, proposed by Derbenev and Kondratenko in 1978 [1], involves using a spin rotator called a Siberian snake that makes the spin tune equal to $1 / 2$ independent of the beam energy. This energy-independent $\nu_{s}$ eliminates most of the depolarizing resonances.

Once a polarized proton beam is accelerated to a high energy and stored, it is important to be able to reverse its polarization direction in order to reduce the systematic error in various polarized scattering asymmetry experiments.

Studies at the IUCF Cooler Ring show that these polarization reversals (spinflips) can be done using an rf magnet [2,3], which creates an rf depolarizing resonance:

$$
f_{s}=f_{r f}+n f_{c}
$$

where $f_{r f}$ is the rf magnet's frequency, $f_{c}$ is the circulation frequency, and $n$ is an integer. After crossing such a resonance, which is varying the rf magnet's frequency from a value below the resonance to a value above the resonance, the final beam polarization $P_{f}$ is related to the initial beam polarization $P_{i}$ via the Froissart-Stora formula [4]:

$$
P_{f}=P_{i}\left[2 e^{-\frac{\left(\pi \in f_{c}\right)^{2}}{(\Delta f / \Delta t)}}-1\right]
$$

where $\epsilon$ is the resonance strength, and $\Delta f / \Delta t$ is the resonance crossing rate, while $\Delta f$ is the frequency's range during its linear variation time $\Delta t$. If the resonance is sufficiently strong and/or the crossing rate is sufficiently slow, the final polarization is reversed with respect to the initial polarization, while its absolute value is the same; this is called spin flip.

\section{EXPERIMENTAL APPARATUS}

The apparatus used for this experiment is shown in Fig. 1. The $120 \mathrm{MeV}$ horizontally polarized proton beam in the Cooler Ring was obtained using the new Cooler Injector Polarized IOn Source (CIPIOS) [5] and the new Cooler Injection Synchrotron (CIS) [6]. Also shown are the Siberian snake solenoid with its eight 
correction quadrupoles, the polarimeter and the rf-dipole magnet, which is one of the Cooler Ring's injection kicker dipoles. In order to increase the strength of the rf-dipole, we connected to it a variable capacitor to form a resonant parallel LCcircuit. The maximum r.m.s. voltage on the rf-dipole that we were able to reach was about $71 \mathrm{~V}$; this corresponded to an $\int B \cdot d l$ of about $0.06 \mathrm{~T} \cdot \mathrm{mm}$.

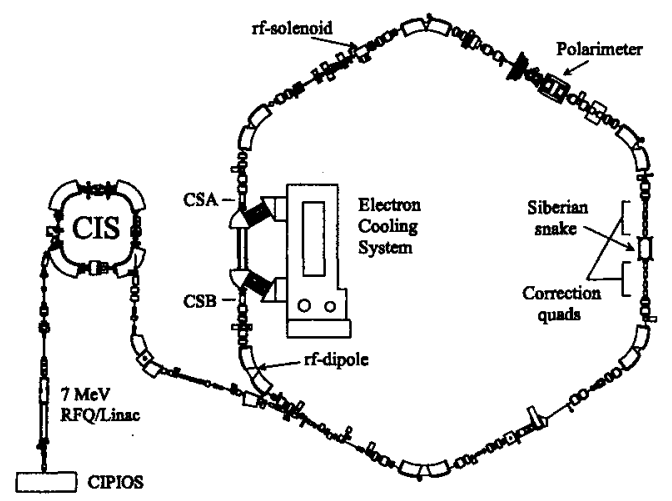

FIGURE 1. IUCF Cooler Ring and its hardware.

\section{RESULTS AND ANALYSIS}

We first determined the rf depolarizing resonance's location by measuring the radial beam polarization at different rf-dipole's frequencies; this measured radial polarization is plotted against the rf-dipole's frequency in Fig. 2 [7]. The curve is a second-order Lorentzian fit to the data with a resonance frequency of $2384040 \pm 90 \mathrm{~Hz}$ and a width $970 \pm 20 \mathrm{~Hz}$.

To study spin-flipping, we first set our frequency ramp range $\Delta f$ to $10 \mathrm{kHz}$

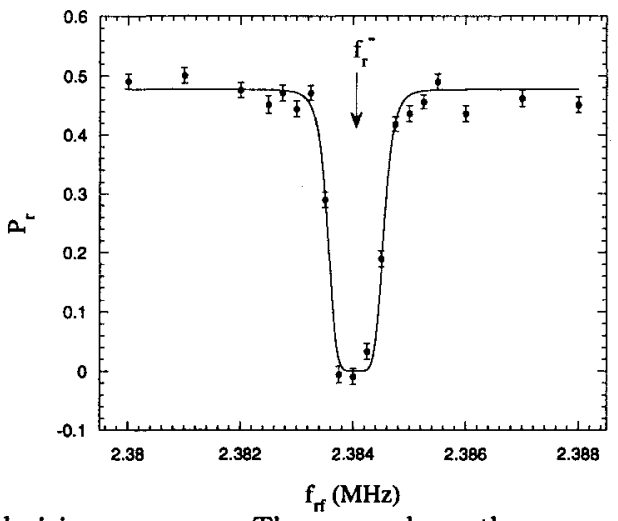

FIGURE 2. RF depolarizing resonance. The arrow shows the resonance's central frequency. 
and crossed this rf-induced resonance by linearly ramping the rf-dipole's frequency from $f_{r}-5$ to $f_{r}+5 \mathrm{kHz}$, with various ramp times $\Delta t$, while measuring the beam polarization after each crossing. Thus, we determined the optimal ramp time for the most efficient spin-flip. We then tried to further increase the spin-flip efficiency by varying the rf-dipole's frequency range $\Delta f$. After setting $\Delta t$ and $\Delta f$ to maximize the spin-flip efficiency, we more precisely determined this efficiency by performing many spin-flips. The measured radial beam polarization after several spin-flips is plotted against the number of spin-flips in Fig. 3. We fit this data using:

$$
P_{n}=P_{i} \cdot \eta^{n}
$$

where $P_{n}$ is the measured radial beam polarization after $n$ spin-flips, $P_{i}$ is the initial polarization, $\eta$ is the spin-flip efficiency, and $n$ is the number of spin-flips. The best fit gave a spin-flip efficiency of $86.4 \pm 0.5 \%$ [7]. The spin-flip efficiency was apparently limited by the strength of the rf-dipole's field.

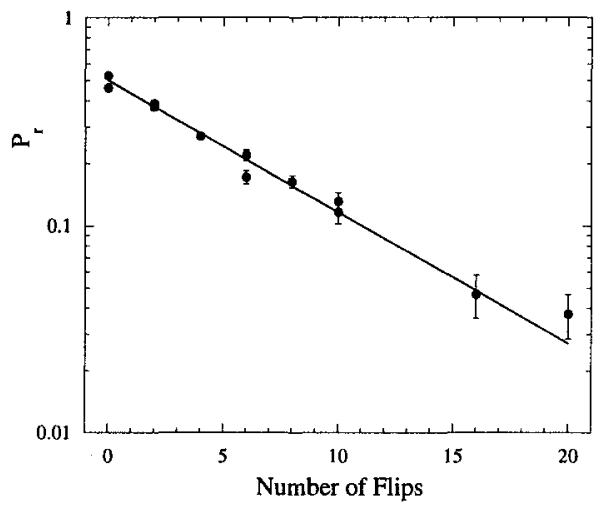

FIGURE 3. Multiple spin-flipping.

In conclusion, we have successfully demonstrated the feasibility of an rf-dipole magnet as a spin-flipper in a storage ring where a full Siberian snake is present. We are currently developing an rf-dipole spin-flipper for the MIT-Bates polarized electron ring.

\section{REFERENCES}

1. Ya. S. Derbenev and A. M. Kondratenko, Part. Accel. 8, 115 (1978).

2. B.B. Blinov et al., Phys. Rev. Lett. 81, 2906 (1998).

3. V. A. Anferov et al. Phys. Rev. ST-AB 3, 041001 (2000).

4. M. Froissart and R. Stora, Nucl. Instrum. and Methods 7, 297 (1960).

5. V.P. Derenchuk and A.S. Belov, in AIP Conf. Proc. 421, 422 (1998).

6. D.L. Friesel and S.Y. Lee, in Proc. of 1997 Particle Accelerator Conf (PAC-97), Vancouver, 935 (1998).

7. B.B. Blinov et al., Submitted to Phys. Rev. Lett. June 2000. 